
Brasileira 27: 091-095.

\title{
Produção de frutos de morango em função de diferentes períodos de vernalização das mudas
}

\author{
Roberto P de Oliveira; Walkyria B Scivittaro \\ Embrapa Clima Temperado, C. Postal 403, 96001-970 Pelotas-RS; rpedroso@cpact.embrapa.br; wbscivit@cpact.embrapa.br
}

\begin{abstract}
RESUMO
Avaliou-se a produção de frutos de morango da cv. Camarosa em função de diferentes períodos de vernalização das mudas, comparando-a com a de mudas importadas. O experimento foi realizado em Pelotas, utilizando sistema de produção sob túnel. O transplante foi realizado em 18/05/06, utilizando-se mudas produzidas no sul do Rio Grande do Sul, vernalizadas por $0 ; 7 ; 14 ; 21$ e 28 dias $\left(4 \pm 1^{\circ} \mathrm{C}, 94 \pm 2 \%\right.$ UR), e mudas não vernalizadas do Chile. Os tratamentos foram dispostos em delineamento de blocos ao acaso, com quatro repetições. A unidade experimental foi constituída por 21 plantas, avaliadas quanto à produção, número de frutos por planta e massa média dos frutos ao longo de 20 semanas (agosto a dezembro de 2006). As mudas chilenas proporcionaram maiores produção e número de frutos do que as produzidas no RS na ausência de vernalização (1.038,3 e 491,7 $\mathrm{g} \mathrm{planta}^{-1} ; 55,1 \mathrm{e}$ 34,3 frutos planta ${ }^{-1}$, respectivamente). A vernalização das mudas nacionais por cerca de 24 dias otimizou a produção e o número de frutos $\left(1.023,1 \mathrm{~g} \mathrm{planta}^{-1} ; 55,6\right.$ frutos planta $\left.^{-1}\right)$. As mudas produzidas no RS apresentaram maior produção em novembro e dezembro, enquanto as chilenas em outubro e novembro.
\end{abstract}

\begin{abstract}
Production of strawberry fruits depending on vernalization periods of the transplants

The yield of strawberry fruits cv. Camarosa was evaluated as affected by different vernalization periods of the transplant, in comparison with imported ones. The experiment was carried out in Pelotas, Rio Grande do Sul State, Brazil, using a tunnel as production system. The plants were transplanted on May18, 2006, using transplants produced in the south of Rio Grande do Sul, which were vernalized during $0 ; 7 ; 14 ; 21$ and 28 days $\left(4 \pm 1^{\circ} \mathrm{C}, 94 \pm 2 \% \mathrm{RH}\right)$, and transplants without vernalization coming from Chile. The treatments were disposed according to a randomized complete block design with four replications. The experimental unit were composed of 21 plants. The yield, number of fruits per plant and average fruit mass were analyzed during 20 weeks (August to December). The Chilean transplants provided greater production and number of fruits than transplants produced in Rio Grande do Sul (1,038.3 and $491.7 \mathrm{~g}$ plant $^{-1} ; 55.1$ and 34.3 fruits plant ${ }^{-1}$, respectively). The vernalization of the national transplants during approximately 24 days optimized the yield and number of fruits significantly $\left(1,023.1 \mathrm{~g} \mathrm{plant}^{-1} ; 55.6\right.$ fruits plant ${ }^{-1}$ ). Transplants produced in Rio Grande do Sul presented higher production in November and December, while Chilean ones in October and November.
\end{abstract}

Keywords: Fragaria x ananassa, 'Camarosa', temperature.

Palavras-chave: Fragaria x ananassa, 'Camarosa', temperatura.

\section{(Recebido em 28 de março de 2007; aceito em 15 de dezembro de 2008) (Received in March 28, 2007; accepted in December 15, 2008)}

$\mathrm{N}$ o Brasil, a cultura do morangueiro encontra-se difundida em regiões de clima temperado e subtropical, onde se produz para consumo in natura e industrialização. A produção de morango destaca-se pela alta rentabilidade por área e demanda intensa de mão-de-obra (Oliveira et al., 2005).

A produção mundial de morango é de 3,1 milhões de toneladas por ano e a brasileira de 37,6 mil toneladas, sendo esta última obtida em uma área estimada de 3,5 mil hectares, com destaque para Minas Gerais (41,4\%), Rio Grande do Sul (25,6\%) e São Paulo (15,4\%) (IEA, 2007).

As principais cultivares de morangueiro utilizadas no Brasil provêm dos Estados Unidos, destacando-se Aromas, Camarosa, Camino Real, Diamante, Dover, Oso Grande, Sweet Charlie e
Ventana. No Rio Grande do Sul, a Camarosa é a cultivar de dias curtos mais plantada, sendo utilizada para consumo in natura e produção de doces, sucos e geléias (Oliveira et al., 2005).

A muda é um dos principais insumos do sistema de produção de morango. A mesma está diretamente relacionada com a produtividade e a qualidade do fruto, sendo a base para uma melhor resposta às tecnologias empregadas no processo produtivo. No Rio Grande do Sul, mais de $80 \%$ das mudas utilizadas pelos agricultores provêm do Chile e da Argentina (Oliveira et al., 2005).

Segundo Durner et al. (1987), Ronque (1998) e Stapleton et al. (2001), a produção do morangueiro está diretamente relacionada com o número de horas de frio que as mudas recebem nos viveiros. É recomendável instalar os viveiros em locais de latitude e/ou altitude elevadas, a fim de que o número de horas acumuladas de frio $\left(2\right.$ a $\left.7^{\circ} \mathrm{C}\right)$ seja de 380 a 700 (Ronque, 1998). Para compensar o menor número de horas de frio de mudas produzidas em viveiros localizados em latitudes médias e baixas, vários autores recomendam a sua vernalização (Brandão Filho et al., 1989; Tehranifar et al., 1998 e Faedi \& Baruzzi, 2002). Ainda não existe relato sobre estudos de vernalização da cultivar Camarosa, comparando a produtividade de mudas nacionais vernalizadas com importadas. Portanto, avaliou-se nesse trabalho o efeito de períodos de vernalização das mudas sobre a produção de frutos de morango da cv. Camarosa. 
Tabela 1. Produção acumulada de frutos comerciais, número de frutos por planta e massa média de frutos de morangueiro da cv. Camarosa colhidos de agosto a dezembro de 2006, em função da procedência e do período de vernalização da muda (production of commercial fruits, number of fruit per plant and average fruit mass of strawberry cv. Camarosa harvested from August to December 2006, according to the source and the period of transplant vernalization). Pelotas, Embrapa Clima Temperado, 2006.

\begin{tabular}{lccc}
\hline $\begin{array}{l}\text { Procedência/ período de } \\
\text { vernalização }\end{array}$ & $\begin{array}{c}\text { Produção } \\
\text { comercial (g } \\
\text { planta }^{-1} \text { ) }\end{array}$ & $\begin{array}{c}\text { Número de frutos } \\
\text { por planta }\end{array}$ & $\begin{array}{c}\text { Massa média dos } \\
\text { frutos (g) }\end{array}$ \\
\hline RS/sem vernalização & $491,7 \mathrm{~d}$ & $34,3 \mathrm{c}$ & $17,0 \mathrm{~b}$ \\
RS/vernalização por 7 dias & $802,5 \mathrm{c}$ & $43,4 \mathrm{~b}$ & $17,8 \mathrm{ab}$ \\
RS/vernalização por 14 dias & $856,8 \mathrm{bc}$ & $50,3 \mathrm{ab}$ & $16,8 \mathrm{~b}$ \\
RS/vernalização por 21 dias & $1.069,6 \mathrm{a}$ & $57,9 \mathrm{a}$ & $19,4 \mathrm{a}$ \\
RS/vernalização por 28 dias & $994,7 \mathrm{~b}$ & $54,1 \mathrm{a}$ & $18,4 \mathrm{ab}$ \\
Chile/sem vernalização & $1.038,3 \mathrm{a}$ & $55,1 \mathrm{a}$ & $19,5 \mathrm{a}$ \\
\hline C.V. (\%) & 7,2 & 7,4 & 4,2 \\
\hline
\end{tabular}

Médias seguidas de mesma letra nas colunas, não diferem significativamente entre si pelo teste de Tukey a 5\% de probabilidade (means followed by the same letter in the columns do not differ according to Tukey's test at the $5 \%$ level of probability).

\section{MATERIAL E MÉTODOS}

De plantas matrizes produzidas na Embrapa Clima Temperado, em Pelotas (latitude $31^{\circ} 46^{\prime} 00^{\prime \prime}$, altitude de $245 \mathrm{me}$ temperatura média anual de $17,6^{\circ} \mathrm{C}$ ) e na região sul do Chile (latitude $37^{\circ} 28^{\prime} 00^{\prime \prime}$; altitude de $139 \mathrm{~m}$ ) foram obtidas mudas comerciais, para instalação do presente experimento, em Pelotas-RS. Inicialmente, as folhas das mudas foram removidas, mantendo-se as raízes intactas, sendo realizado um tratamento preventivo com o fungicida tiofanato metil $\left(2 \mathrm{~g} \mathrm{~L}^{-1}\right)$.

Os tratamentos consistiram de mudas não vernalizadas produzidas na região de Pelotas (RS/sem vernalização); mudas vernalizadas por $7 ; 14 ; 21$ e 28 dias produzidas na região de Pelotas (RS/vernalização); e mudas não vernalizadas produzidas no Chile (Chile/sem vernalização).

A vernalização das mudas foi realizada em câmara fria à temperatura de $4 \pm 1^{\circ} \mathrm{C}$ e umidade relativa de $94 \pm 2 \%$. Durante o tratamento de vernalização, as mudas permaneceram no interior de sacos plásticos transparentes de $0,05 \mathrm{~mm}$ de espessura, contendo 21 plantas cada.

$\mathrm{O}$ transplante das mudas foi realizado em 18/05/06, em canteiros de $1,2 \mathrm{~m}$ de largura por $0,15 \mathrm{~m}$ de altura, espaçados entre si em 0,8 m, em solo corrigido (Sociedade, 2004). O espaçamento entre linhas e entre plantas foi de $0,35 \mathrm{~m}$, sendo utilizadas três linhas por canteiro. Os canteiros foram revestidos com filme de polietileno preto com 30 micras de espessura.

Utilizou-se o sistema de produção em túneis com $0,5 \mathrm{~m}$ de altura na parte central, sustentados por arcos de arame galvanizado e cobertos com filme de polietileno transparente aditivado, com 100 micras de espessura.

Os seis tratamentos foram distribuídos em delineamento experimental de blocos casualizados, com quatro repetições. Cada parcela experimental foi composta por 21 plantas de morangueiro.

As plantas de morangueiro foram irrigadas por gotejamento, diariamente, de acordo com a necessidade hídrica. O controle das plantas daninhas, a remoção de folhas secas e com sintomas de doenças, de estolões e de frutos danificados ou com defeitos foi feito manualmente. Semanalmente, as plantas foram fertirrigadas, com aplicações alternadas dos fertilizantes $\mathrm{KSCl}^{\circledast}\left(14 \% \mathrm{~N}, 40 \% \mathrm{P}_{2} \mathrm{O}_{5}, 5 \% \mathrm{~K}_{2} \mathrm{O}, 13 \% \mathrm{~S}\right.$, $0,1 \% \mathrm{~B}, 0,05 \% \mathrm{Cu}, 0,1 \% \mathrm{Fe}, 0,01 \%$ Mo e $0,1 \% \mathrm{Zn}), \mathrm{KSC}^{\oplus}\left(8 \% \mathrm{~N}, 16 \% \mathrm{P}_{2} \mathrm{O}_{5}, 42 \%\right.$ $\mathrm{K}_{2} \mathrm{O}, 0,1 \% \mathrm{~B}, 0,1 \% \mathrm{Fe}, 0,05 \%$ Mn e $0,1 \%$ $\mathrm{Zn}$ ) e nitrato de cálcio (500 g por 1000 plantas). O tratamento fitossanitário incluiu duas aplicações com iprodione $(150 \mathrm{~mL}$ por $100 \mathrm{~L})$ e uma com azoxystrobin $(15 \mathrm{~g}$ por $100 \mathrm{~L}$ ), para o controle de doenças fúngicas, uma aplicação com abamectina (50 mL por $100 \mathrm{~L})$, para o controle de ácaros, e a utilização de iscas para o controle de broca das frutas (Lobiopa insularis) e de camundongos.
O período de colheita dos frutos, considerado para a avaliação do experimento, estendeu-se do início do mês de agosto ao final do mês de dezembro de 2006. Ao longo desse período, determinaram-se, semanalmente, a massa fresca e o número de frutos produzidos por unidade experimental. A massa média dos frutos foi calculada pela razão entre essas duas variáveis. Foram determinados ainda, o número e a massa média acumulada dos frutos por planta, bem como a produção acumulada, considerando-se o período integral de colheita (20 semanas). Para a avaliação das características acima, foram considerados apenas os frutos comercializáveis, descartando-se aqueles com defeitos, em deterioração e com massa inferior a $3 \mathrm{~g}$.

Os dados relativos ao período integral de colheita foram submetidos à análise de variância, comparando-se as médias dos tratamentos pelo teste Tukey $(\mathrm{p} \leq 0,05)$. Adicionalmente, os dados semanais de colheita foram reunidos em grupos de quatro semanas, perfazendo cinco períodos de avaliação, os quais corresponderam às colheitas dos meses de agosto, setembro, outubro, novembro e dezembro. Estes foram analisados considerando-se delineamento de blocos casualizados com parcelas subdivididas no tempo. As médias dos fatores tratamento e período de avaliação foram analisadas pelo teste Tukey $(\mathrm{p} \leq 0,05)$. $\mathrm{O}$ efeito do período de vernalização das mudas nacionais foi avaliado por meio de análise de regressão polinomial, considerando-se os dados relativos ao período total de colheita.

\section{RESULTADOS E DISCUSSÃO}

Ao longo dos cinco meses de colheita, as mudas importadas do Chile apresentaram melhor desempenho do que aquelas produzidas no sul do Rio Grande do Sul na ausência de vernalização, quanto à produção acumulada de frutos comerciais, número e massa média dos frutos (Tabela 1). O desempenho superior das mudas importadas em relação às nacionais também foi observado por Oliveira \& Scivittaro (2006). Segundo Durner et al. (1987), Ronque (1998) e Stapleton et al. (2001), a produção do morangueiro está direta- 
mente relacionada ao número de horas de frio que as mudas recebem nos viveiros. Para Ronque (1998), recomenda-se que os viveiros estejam localizados em regiões de latitude e/ou altitude elevadas, a fim de que o número de horas acumuladas de frio $\left(2\right.$ a $\left.7^{\circ} \mathrm{C}\right)$ seja de 380 a 700. Desta forma, a maior produtividade das plantas oriundas de mudas do Chile (latitude 37²8'00") em relação às do Rio Grande do Sul (latitude 31 46'00”) pode ser atribuída ao maior número de horas de frio recebido durante o período de formação da muda. Além disso, as mudas chilenas são armazenadas e transportadas para o Brasil em caminhões frigoríficos, simulando uma vernalização, ao passo que as mudas nacionais são geralmente transplantadas quase imediatamente após o arranquio (Santos \& Medeiros, 2003). Stapleton et al. (2001), utilizando a cultivar de morangueiro Sweet Charlie, também obtiveram maior produção de frutos em plantas provenientes de região de maior latitude $(23,1$ $\mathrm{t} \mathrm{ha}^{-1}$ ) em relação à de menor latitude (18,1 tha $\left.^{-1}\right)$.

Com relação ao efeito do período de vernalização das mudas nacionais, determinou-se significância para as variáveis produção acumulada de frutos comerciais, número de frutos e massa média de frutos. Para as duas primeiras, os dados ajustaram-se a modelos quadráticos, com valores máximos correspondentes aos períodos de 24,2 e 24,6 dias, respectivamente (Figuras 1 e 2). Estes resultados demonstram a importância da vernalização para a muda nacional otimizando a produção e conferindo competitividade com o mercado de mudas chilenas. Por sua vez, os dados de massa média dos frutos ajustaram-se ao modelo linear crescente: $y=16,995+$ $0,0643 x ; R^{2}=0,43 * *$. Os dados relativos a esta variável indicam, ainda, menor sensibilidade ao efeito do período de vernalização, relativamente às variáveis produção e número de frutos de morango produzidos.

Ao se comparar as mudas produzidas no Rio Grande do Sul sem e com vernalização por 21 dias, verifica-se maior produção acumulada (1069,6 g planta $\left.^{-1}\right)$ e número de frutos produzidos por planta $(57,9)$ para as plantas provenientes de mudas vernalizadas, o que representou incremento de $117 \%$ e $69 \%$,

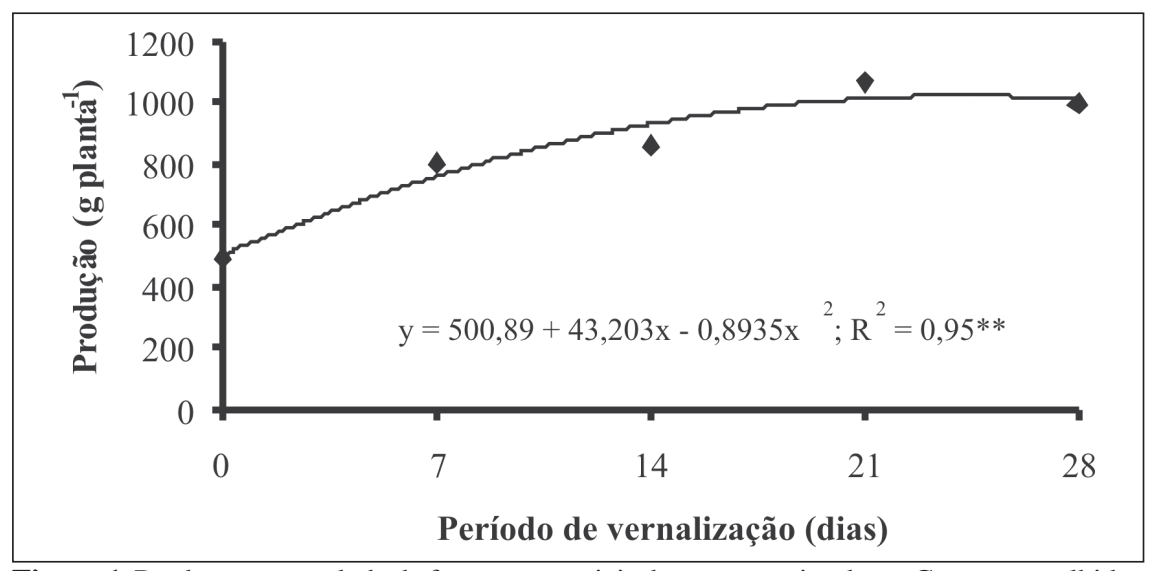

Figura 1. Produção acumulada de frutos comerciais de morangueiro da cv. Camarosa colhidos de agosto a dezembro de 2006, em função do período de vernalização da muda produzida no Rio Grande do Sul (Production of commercial fruits of strawberry cv. Camarosa harvested from August to December 2006, according to the vernalization period of the transplant produced in Rio Grande do Sul). Pelotas, Embrapa Clima Temperado, 2006.

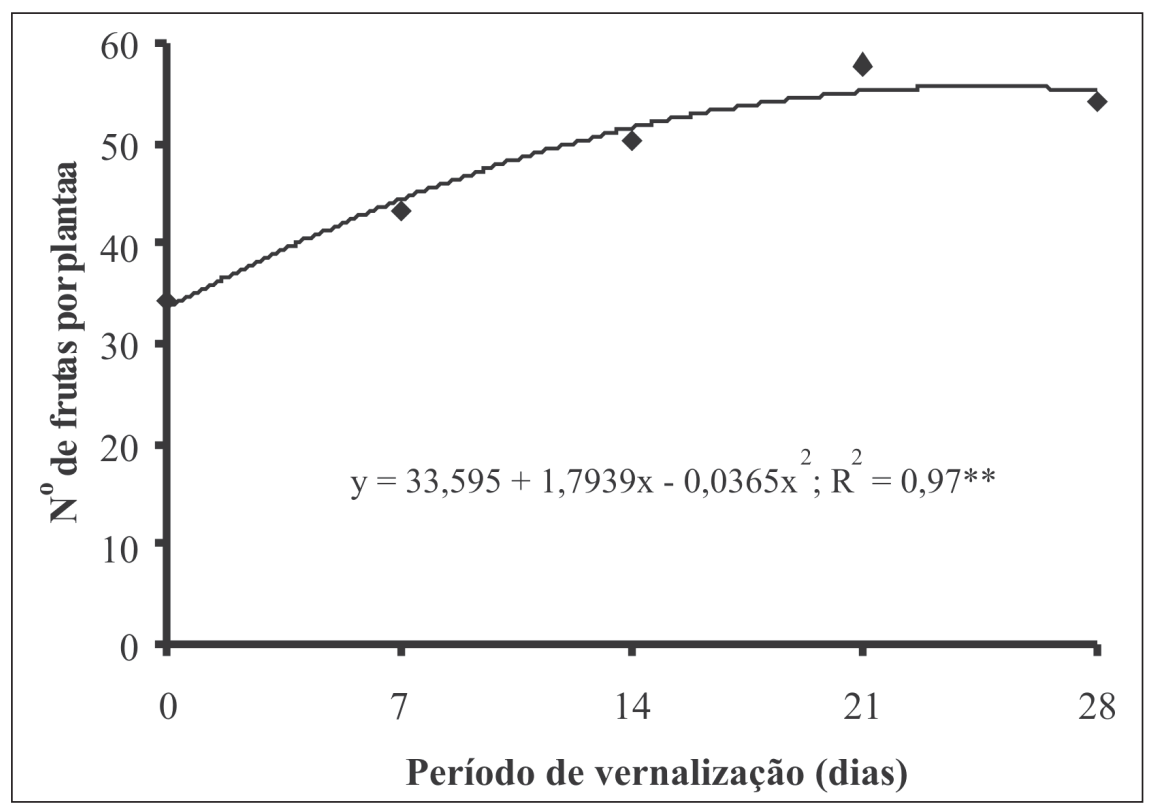

Figura 2. Número médio de frutos produzidos por planta de morangueiro da cv. Camarosa colhidos de agosto a dezembro de 2006, em função do período de vernalização da muda produzida no Rio Grande do Sul (average number of fruits produced per plant of strawberry cv. Camarosa harvested from August to December 2006, according to the vernalization period of the transplant produced in Rio Grande do Sul). Pelotas, Embrapa Clima Temperado, 2006.

respectivamente (Tabela 1 ). $\mathrm{O}$ benefício da vernalização de mudas de morangueiro também foi descrito por Brandão Filho et al. (1989), Tehranifar et al. (1998) e Faedi \& Baruzzi (2002). Na Itália, estes últimos autores reportaram aumento de $30 \%$ na produção de morango, oriunda de mudas vernalizadas.

A vernalização da muda nacional por 21 dias conferiu-lhe potencial de produção semelhante ao da muda chilena (Tabela 1). Rice Jr. (1990), não verificou efeito do período de vernalização das mudas na produção de frutos de morangueiro da cultivar Tioga, mantida a $5^{\circ} \mathrm{C}$. No entanto, obteve maior produção ao vernalizar mudas de morangueiro 'Selekta' e 'Rovelle' por sete dias. Tehranifar et al. (1998) obtiveram maior produção de frutos da cv. Elsanta ao armazenarem as mudas por 28 dias em câmara fria a $3^{\circ} \mathrm{C}$.

Nos dois primeiros meses de colheita (agosto e setembro) não houve efeito da procedência e do período de vernalização da muda na produção e no 
Tabela 2. Produção de frutos comerciais $\left(g\right.$ planta $\left.{ }^{-1}\right)$ de morangueiro da cv. Camarosa, em função da procedência, período de vernalização da muda e época de colheita (production of commercial fruits $\left(\mathrm{g}\right.$ plant ${ }^{-1}$ ) of strawberry cv. Camarosa, according to the source, period of transplant vernalization and month of harvest). Pelotas, Embrapa Clima Temperado, 2006.

\begin{tabular}{|c|c|c|c|c|c|}
\hline \multirow{2}{*}{$\begin{array}{l}\text { Procedência/período de } \\
\text { vernalização }\end{array}$} & \multicolumn{5}{|c|}{ Época de colheita } \\
\hline & Agosto & Setembro & Outubro & Novembro & Dezembro \\
\hline RS/sem vernalização & $49,5 a$ B & $59,9 a \mathrm{~B}$ & $118,0 \mathrm{c} \mathrm{A}$ & $116,9 \mathrm{~d} A$ & $147,4 \mathrm{~d} \mathrm{~A}$ \\
\hline RS/vernalização por 7 dias & $34,4 a \mathrm{D}$ & $55,6 a \mathrm{D}$ & $174,2 b \mathrm{C}$ & 245,5 c B & $292,8 b$ A \\
\hline RS/vernalização por 14 dias & $47,2 \mathrm{a} C$ & $64,6 a \mathrm{C}$ & $172,0 \mathrm{~b} \mathrm{~B}$ & $281,4 \mathrm{c} \mathrm{A}$ & $291,6 b$ A \\
\hline RS/vernalização por 21 dias & 22,7 a C & $40,0 \mathrm{a} C$ & $165,0 \mathrm{bc} \mathrm{B}$ & $438,9 a \mathrm{~A}$ & $403,1 \mathrm{a} \mathrm{A}$ \\
\hline RS/vernalização por 28 dias & 28,7 a C & $52,0 \mathrm{a} C$ & $178,8 \mathrm{~b} \mathrm{~B}$ & $370,0 \mathrm{~b} \mathrm{~A}$ & $365,1 \mathrm{a} \mathrm{A}$ \\
\hline Chile/sem vernalização & $65,3 a \mathrm{C}$ & $58,2 a \mathrm{C}$ & $348,6 a$ A & $355,4 \mathrm{~b} \mathrm{~A}$ & 210,7 c B \\
\hline
\end{tabular}

Médias seguidas de mesma letra, minúscula nas colunas e maiúscula nas linhas, não diferem significativamente entre si pelo teste de Tukey a 5\% de probabilidade (means followed by the same lowercase letter in the columns and by the uppercase letter in the rows do not differ according to Tukey's test at the 5\% level of probability).

Tabela 3. Número e massa média (g) de frutos produzidos por planta de morangueiro da cv. Camarosa, em função da procedência, período de vernalização da muda e época de colheita (number of fruits per plant and average fruit mass ( $\mathrm{g}$ ) of strawberry cv. Camarosa, according to the source, period of transplant vernalization and month of harvest). Pelotas, Embrapa Clima Temperado, 2006.

\begin{tabular}{lccccc}
\hline \multirow{2}{*}{$\begin{array}{l}\text { Procedência / } \\
\text { Período de vernalização }\end{array}$} & \multicolumn{5}{c}{ Época de colheita } \\
\cline { 2 - 6 } & Agosto & Setembro & Outubro & Novembro Dezembro \\
\cline { 2 - 6 } RS/sem vernalização & $3,7 \mathrm{aB}$ & $4,2 \mathrm{aB}$ & $3,6 \mathrm{cB}$ & $12,4 \mathrm{eA}$ & $10,4 \mathrm{eA}$ \\
RS/vernalização por 7 dias & $2,6 \mathrm{aD}$ & $3,8 \mathrm{aD}$ & $7,0 \mathrm{bC}$ & $13,2 \mathrm{deB}$ & $16,9 \mathrm{cdA}$ \\
RS/vernalização por 14 dias & $3,2 \mathrm{aD}$ & $4,6 \mathrm{aD}$ & $7,9 \mathrm{bC}$ & $15,8 \mathrm{cdB}$ & $23,7 \mathrm{bA}$ \\
RS/vernalização por 21 dias & $1,3 \mathrm{aD}$ & $2,7 \mathrm{aD}$ & $6,0 \mathrm{bcC}$ & $21,1 \mathrm{aB}$ & $26,7 \mathrm{aA}$ \\
RS/vernalização por 28 dias & $1,8 \mathrm{aD}$ & $3,7 \mathrm{aD}$ & $6,7 \mathrm{bC}$ & $18,2 \mathrm{bcB}$ & $23,7 \mathrm{bA}$ \\
Chile/sem vernalização & $3,6 \mathrm{aD}$ & $2,9 \mathrm{aD}$ & $12,1 \mathrm{aC}$ & $20,7 \mathrm{abA}$ & $15,8 \mathrm{~dB}$ \\
\hline C.V. (procedência/período vernalização) $=7,4 \%$ & $\mathrm{C} . \mathrm{V}$. (época de colheita) $=13,0 \%$ \\
\hline & \multicolumn{5}{c}{ Massa média do fruto (g) } \\
\hline RS/sem vernalização & $13,5 \mathrm{bB}$ & $14,3 \mathrm{bB}$ & $33,3 \mathrm{aA}$ & $9,4 \mathrm{bC}$ & $14,3 \mathrm{abB}$ \\
RS/vernalização por 7 dias & $13,2 \mathrm{bD}$ & $14,8 \mathrm{bCD}$ & $25,1 \mathrm{bcA}$ & $18,7 \mathrm{aB}$ & $17,4 \mathrm{aBC}$ \\
RS/vernalização por 14 dias & $15,0 \mathrm{abBC}$ & $14,0 \mathrm{bC}$ & $21,8 \mathrm{cA}$ & $17,8 \mathrm{aB}$ & $15,4 \mathrm{abBC}$ \\
RS/vernalização por 21 dias & $17,6 \mathrm{aBC}$ & $14,7 \mathrm{bC}$ & $27,6 \mathrm{bA}$ & $20,7 \mathrm{aB}$ & $15,1 \mathrm{abC}$ \\
RS/vernalização por 28 dias & $15,6 \mathrm{abC}$ & $14,0 \mathrm{bC}$ & $26,9 \mathrm{bA}$ & $20,7 \mathrm{aB}$ & $15,4 \mathrm{abC}$ \\
Chile/sem vernalização & $18,6 \mathrm{aB}$ & $19,6 \mathrm{aB}$ & $28,8 \mathrm{bA}$ & $17,1 \mathrm{aBC}$ & $13,4 \mathrm{bC}$ \\
\hline C.V. (procedência/período vernalização) $=4,2 \%$ & C.V. (época de colheita) = 10,8\% \\
\hline
\end{tabular}

Médias seguidas de mesma letra, minúscula nas colunas e maiúscula nas linhas, não diferem significativamente entre si pelo teste de Tukey a 5\% de probabilidade (means followed by the same lowercase letter in the columns and by the uppercase letter in the rows do not differ according to Tukey's test at the 5\% level of probability).

número de frutos produzidos por planta. Nos demais meses, de modo geral, o contrário aconteceu para as variáveis analisadas (Tabelas 2 e 3 ). As mudas procedentes do Chile proporcionaram maior produção nos meses de outubro e novembro, enquanto que as produzidas no Rio Grande do Sul em novembro e dezembro (Tabela 2). Desta forma, as mudas do Chile são indicadas para os concentrar-se no meio do ano (Agrianual, 2006). A utilização de mudas de ambas as procedências é interessante para os produtores que desejam escalonar a produção, principalmente para otimizar o uso da mão-de-obra, altamente demandada na cultura do morangueiro. Os resultados apresentados são concordantes com aqueles reportados por Durner et al. (1987) e Stapleton et al. (2001), segundo os quais as mudas produzidas sob latitudes maiores produzem precocemente. A redução na produção das plantas oriundas de mudas importadas do Chile no mês de dezembro deve-se ao fato de a cultivar Camarosa ser de dias curtos, sendo, comumente, mais produtiva na primavera, com diminuição da produção à medida que as temperaturas médias vão aumentando e os dias se tornando mais longos (Santos \& Medeiros, 2003).

Independentemente da procedência e do período de vernalização, as plantas de morangueiro apresentaram maior massa média de frutos no mês de outubro, com variação de 21,8 a 33,3 g (Tabela 3). Observa-se que, embora tenham ocorrido diferenças significativas na massa dos frutos entre os tratamentos, estas variaram entre os meses de colheita. Este comportamento é explicado pelo fato de que quando a produção é elevada, existe uma tendência de se produzir frutos de menor tamanho (Domingues et al., 2001).

Os valores de massa média dos frutos obtidos ao longo do período de colheita (9,4 a 33,3 g) (Tabela 3), foram superiores ao patamar de comercialização estabelecido por Souza (1972) para morango tipo especial ( 8 a $14 \mathrm{~g}$ ), revelando o potencial da cultivar Camarosa para a produção de frutos grandes (University of California, 2006).

Com base nos resultados obtidos, pode-se concluir que: 1) As mudas chilenas proporcionam maior produção e número de frutos por planta do que as mudas produzidas no Rio Grande do Sul na ausência de vernalização; 2) A vernalização das mudas nacionais por cerca de 24 dias otimiza a produção e o número de frutos produzidos por planta. 3) As mudas produzidas no Rio Grande do Sul apresentam maior produção em novembro e dezembro, enquanto as chilenas em outubro e novembro. 


\section{AGRADECIMENTOS}

À FAPERGS e ao CNPq pelo financiamento do projeto e concessão de bolsas.

\section{REFERÊNCIAS}

AGRIANUAL - Anuário da Agricultura Brasileira. 2006. 11. ed. São Paulo: FNP Consultoria e AgroInformativos. 504p.

BRANDÃO FILHO JUT; IKUTA J; ISHIMURA I; IZIOJA H; NARITA N; ODA N; TARGINO A; KIMOTO T. 1989. Efeito do tempo de vernalização sobre o florescimento do morangueiro. Horticultura Brasileira 17: 45-46.

DOMINGUES MCS; ONO EO; RODRIGUES JD. 2001. Reguladores vegetais e o desbaste químico de frutos de tangor Murcote. Scientia Agricola 58: 487-490.

DURNER EF; POLING EB; ALBREGTS EA. 1987. Early season yield responses of selected strawberry cultivars to photoperiod and chilling in a Florida winter production system. Journal of the American Society for Horticultural Science 112: 53-56.
FAEDI W; BARUZZI G. 2002. Innovazioni nelle tecniche di coltivazione della fragola. Rivista di Frutticoltura e di Ortofloricultura 64: 19-26.

IEA - Instituto de Economia Agrícola. 2007, 11 de janeiro. Pólos de produção do morango. Disponível em http:// w w w. i e a.s p.gov. b r / o u t / vertexto.php?codtexto $=11 /$

OLIVEIRA RP; SCIVITTARO WB. 2006. Desempenho produtivo de mudas nacionais e importadas de morangueiro. Revista Brasileira de Fruticultura 28: 520-522.

OLIVEIRA RP; NINO AFP; SCIVITTARO WB. 2005. Mudas certificadas de morangueiro: maior produção e melhor qualidade da fruta. A Lavoura 108: 35-38.

RICE JR RP. 1990. Effects of cultivar and environment interactions on runner production, fruit yield, and harvest timing of strawberry (Fragaria $\mathrm{x}$ ananassa) in Zimbabwe. Acta Horticulturae 279: 327-332.

RONQUE ERV. 1998. Cultura do morangueiro; revisão e prática. Curitiba: Emater. 206p.

SANTOS AM; MEDEIROS ARM. 2003. Produção de mudas comerciais. In: SANTOS AM; MEDEIROS, ARM (eds). Morango; produção. Brasília: Embrapa Informação Tecnológica. p. 35-38. (Embrapa Informação Tecnológica. Frutas do Brasil, 40).
SOCIEDADE BRASILEIRA DE CIÊNCIA DO SOLO. 2004. Comissão de Química e Fertilidade do Solo. Manual de adubação e calagem para os Estados do Rio Grande do Sul e de Santa Catarina. 10. ed. Porto Alegre: SBCS/CQFS. 400p.

SOUZA EF. 1972. O morango e sua padronização: classificação de produtos. Gleba 16: 6-8.

STAPLETON SC; CHANDLER CK; LEGARD DE; PRICE JF; SUMLER JUNIOR JC. 2001. Transplant source affects fruiting performance and pests of 'Sweet Charlie' strawberry in Florida. Hort Technology 11: 61-65.

TEHRANIFAR A; MIERE P; BATTEY NH. 1998. The effects of lifting date, chilling duration and forcing temperature on vegetative growth and fruit production in the June bearing strawberry cultivar Elsanta. Journal of Horticultural Science \& Biotechnology 73: 453-460.

UNIVERSITY OF CALIFORNIA. 2006, 03 de março. Strawberry. Disponível em http:/ /www.ucop.edu/ott/strawberry/ 